Mens

revue d'histoire intellectuelle de l'Amérique française

MENS

\title{
Esdras Minville et le nationalisme économique, 1923-1939
}

\section{Dominique Foisy-Geoffroy}

Volume 1, numéro 1, automne 2000

URI : https://id.erudit.org/iderudit/1024439ar

DOI : https://doi.org/10.7202/1024439ar

Aller au sommaire du numéro

\section{Éditeur(s)}

Centre de recherche en civilisation canadienne-française

\section{ISSN}

1492-8647 (imprimé)

1927-9299 (numérique)

Découvrir la revue

Citer cet article

Foisy-Geoffroy, D. (2000). Esdras Minville et le nationalisme économique, 1923-1939. Mens, 1(1), 51-68. https://doi.org/10.7202/1024439ar

\section{Résumé de l'article}

Esdras Minville (1896-1975), économiste, sociologue et penseur nationaliste québécois de la première moitié du $\mathrm{XX}^{\mathrm{e}}$ siècle, professeur puis directeur de l'École des Hautes Études commerciales de Montréal, était un écrivain prolifique qui a produit une oeuvre de haut calibre intellectuel. Une des principales facettes de celle-ci est la tentative d'accorder le nationalisme et l'économique dans une synthèse forte appuyant tout un programme de réformes visant à améliorer le sort de la nation canadienne-française. Cet article passe en revue tant les fondements théoriques de la pensée minvillienne concernant les liens qui unissent le national à l'économique (notions de vie collective, de nation et de nationalisme économique) que les réformes concrètes proposées en vue de restaurer « l'organisme économique et social » canadien-français, soit la décentralisation de la population

(colonisation), de l'activité économique (modernisation de l'agriculture, réseau de petites et de moyennes entreprises) ainsi que de l'exercice du pouvoir étatique (corporatisme).
Ce document est protégé par la loi sur le droit d'auteur. L'utilisation des services d’Érudit (y compris la reproduction) est assujettie à sa politique d'utilisation que vous pouvez consulter en ligne.

https://apropos.erudit.org/fr/usagers/politique-dutilisation/ 


\title{
ESDRAS MINVILLE ET LE NATIONALISME ÉCONOMIQUE, 1923-1939'1
}

\author{
DOMINIQUE FOISY-GEOFFROY \\ Candidat à la maitrise \\ Département d'histoire \\ Université de Montréal
}

\section{RÉSUMÉ}

Esdras Minville (1896-1975), économiste, sociologue et penseur nationaliste québécois de la première moitié du XX' siècle, professeur puis directeur de l'École des Hautes Études commerciales de Montréal, était un écrivain prolifique qui a produit une oeuvre de haut calibre intellectuel. Une des principales facettes de celle-ci est la tentative d'accorder le nationalisme et l'économique dans une synthèse forte appuyant tout un programme de réformes visant à améliorer le sort de la nation canadienne-française. Cet article passe en revue tant les fondements théoriques de la pensée minvillienne concernant les liens qui unissent le national à l'économique (notions de vie collective, de nation et de nationalisme économique) que les réformes concrètes proposées en vue de restaurer (l'organisme économique et sociab) canadien-français, soit la décentralisation de la population (colonisation), de l'activité économique (modernisation de l'agriculture, réseau de petites et de moyennes entreprises) ainsi que de l'exercice du pouvoir étatique (corporatisme).

\section{ABSTRACT}

Principal of Montreal's École des Hautes Études commerciales, sociologist, economist and major French Canadian nationalist intellectual of the first balf of the twentieth century, Esdras Minville (1896-1975) was a prolific and talented writer. As an intellectual, most of bis energy was devoted to the integration of economic thought into nationalism. In this, Minville sought to formulate a Catholic, reform-minded program, bent on improving the economic and moral condition of the French Canadian nation. This article focuses on the theoretical framework of his ideology. Specifically, it examines the aspects that concern the intimate relationship between economics and nationalism present in his thought; on his understanding of collective existence, of the concept of nation and of economic nationalism, and on the reforms be proposed to restore "l'organisme économique et social" of French Canada. This reformist agenda was centred on a program of radical demographic, economic and State decentralization. Decentralization and the devolution of the State's power were to transform Quebec's society through the medium of elaborate agricultural settlement schemes, the development of small and medium-sized industry, and corporatism. 
Esdras Minville était un économiste et un sociologue faisant partie des intellectuels canadiens-français de la mouvance traditionaliste. Professeur à l'École des Hautes Études commerciales de 1924 à 1938, puis directeur de l'institution de 1938 à 1962, fréquentant les milieux nationalistes de son temps, il a laissé une oeuvre importante qui, bien qu'en bonne partie disséminée dans de nombreuses publications, n'en conserve pas moins une cohérence et une richesse qui méritent qu'on s'y arrête. L'École des Hautes Études commerciales, l'École sociale populaire et ses Semaines sociales, les revues $L$ 'Action française, $L ' A c$ tion nationale et L'Actualité économique (dont il est co-fondateur) sont autant de tribunes où, article par article, conférence après conférence, il exposa les éléments d'un programme de restauration économique, sociale et nationale. Il conviait en effet ses compatriotes à la réalisation de ce vaste programme qui, par le redressement de la situation économique de la nation canadienne-française, visait son épanouissement culturel et moral, et, par delà, le salut de la personne.

Pour qui veut étudier les rapports entre le nationalisme et l'économique dans le Québec des années trente, la pensée d'Esdras Minville s'impose parce que ce dernier était considéré comme une autorité tant dans le domaine économique que chez les nationalistes. C'est précisément à une telle étude que cet article est consacré. Nous nous penchons dans ce texte sur la pensée du Minville de l'époque de la Crise, le jeune Minville à ce moment professeur et infatigable chroniqueur de $L ' A c$ tualité économique (son «daboratoire», selon l'expression de François-Albert Angers), où il développa ses thèses. Nous tâcherons donc, dans la première partie du texte, de bien cerner les concepts de vie collective, de nation et de nationalisme économique chez Minville. Quant à la seconde, nous y présenterons globalement le grand programme de réformes qu'il a élaboré en majeure partie pendant la Crise.

\section{Positions théoriques}

La première chose qu'il faut savoir est que les fondements théoriques de la pensée minvillienne n'ont pas réellement été systématisés et organisés jusqu'à la publication d'Invitation à l'étude ${ }^{2}$. Durant la période qui nous intéresse, on peut dire d'une façon générale que Minville ne les fait intervenir que sporadiquement, par fragments disséminés ici et là dans ses écrits, au gré de ses besoins. On trouvera par exemple les bases d'une définition de la nation en introduction d'un texte portant sur l'éducation nationale ou sur la nécessité de tenir compte du facteur national dans la formulation d'une politique économique québécoise. 
Le tableau qui suit présente donc l'interprétation, élaborée à partir de ces fragments, qui nous semblait la plus plausible.

\section{a) Vie collective}

Chez Minville, la vie collective se présente sous différents aspects hiérarchisés et interdépendants, soit, dans l'ordre : le religieux, le moral, le politique, le social et l'économique, qui ferme la marche ${ }^{3}$. Ce qu'il importe notamment de retenir de cette structure de la vie collective, c'est l'interdépendance des différents aspects qui la composent, ce qui, de l'avis de Lionel Groulx, est une des idées directrices de l'oeuvre de Minvillet. Une conséquence importante de ce principe d'interdépendance est qu'on ne peut aborder un aspect de la vie collective sans marquer ses relations avec les autres aspects, au risque de présenter une image faussée et tronquée de la réalité ${ }^{5}$. Par exemple, l'industrialisation, qui est un phénomène économique, a une immense portée sociale et politique, et va jusqu'à conditionner, par ce jeu d'interactions, la quête du salut des hommes. C'est bien là l'essentiel de cette idée d'interdépendance : celle-ci fait le lien entre des activités dont la fin est strictement temporelle, comme l'économique, et les plus hautes destinées de l'homme, intellectuelles, morales, et surtout spirituelles. Les premières sont présentées comme une condition à la réalisation des secondes. Il écrit en effet qu' '[o]n ne saurait concevoir de vie religieuse hors de l'homme avec tous les besoins matériels auxquels celui-ci est assujetti $\left.{ }^{6}{ }^{6}.\right)$ Nous pourrions même aller jusqu'à dire que c'est cette idée d'interdépendance qui donne toute leur valeur aux activités temporelles, et c'est probablement ce qu'entendait Minville lorsqu'il affirmait que l'économique n'est important que dans la mesure où il permet d'atteindre un idéal plus haut? ${ }^{7}$ d'assurer le plein épanouissement de la personnalité de l'individu dans le but de le mettre en état de mieux " servir ${ }^{8}$ ». Ce faisant, Minville reconnaissait et intégrait à son échafaudage théorique que le bien-être matériel est une condition sine qua non de la réalisation des aspirations intellectuelles, morales et spirituelles les plus profondes de l'homme.

Selon ce que nous avons pu dégager de l'oeuvre de Minville, la vie collective, pour lui, est une, et elle est formée de deux composantes principales, soit, d'une part, l'Église, ou la communauté universelle des chrétiens, et, d'autre part, la «cité terrestre». Chacune poursuit sa fin propre correspondant aux plus hautes aspirations de l'homme, et se subordonne tel ou tel aspect de la vie collective, en fonction de sa finalité. Ainsi, la communauté des chrétiens a pour objet le salut de 
l'homme, donc la fin suprême de celui-ci, qui est spirituelle ${ }^{9}$, tandis que la cité s'attacherait les aspects qui concernent plus spécifiquement le bien commun ${ }^{10}$, la fin la plus élevée de l'ordre temporel" ${ }^{11}$.

Ce que Minville appelle la «cité terrestre», ou la "patrie», pourrait elle-même être divisé en trois secteurs correspondant aux trois composantes principales de la vie collective terrestre, soit, premièrement, la société civile ${ }^{12}$, deuxièmement, l'État, ou société politique, et, troisièmement, la nation ou société culturelle $e^{13}$. L'emploi des termes "patrie» et «cité terrestre» est quelque peu ambigu, car ils ne renvoient pas toujours à la même réalité. Cité terrestre, d'une part, semble parfois correspondre à la société civile. Minville fait par exemple souvent référence aux devoirs sociaux et nationaux des Canadiens français, et nous donne ainsi l'impression qu'il distingue bien société et nation. Ici, il parle de devoirs "sociaux et patriotiques "14", là, il nous dit que de l'économique, on débouche sur le social et le nationa ${ }^{15}$. Ailleurs, Minville écrit que le bien commun de la cité pourrait s'appliquer à la nationalité, surtout qu'au Québec, les deux se confondraient à peu près ${ }^{16}$. Dans ce cas précis, la cité semble être associée au Québec, mais en d'autres occasions, Minville parait plutôt faire référence au Canada tout entier, par exemple lorsqu'il parle des responsabilités des Canadiens français envers le «pays ${ }^{17}$ ». En 1927, il écrivait que le Québec est «notre véritable patrie $e^{18}$ ). Dans ces occasions, "cité terrestre» et "patrie» semblent plutôt renvoyer à la société politique, soit canadienne, soit québécoise. Finalement, le terme "patrie» et les mots qui en dérivent sont parfois employés dans le sens de «nation ${ }^{19}$ ». On aura remarqué qu'avec les différents sens que prennent les vocables, on retrouve les trois principales composantes de la cité terrestre signalées précédemment.

C'est ici qu'intervient le principe de l'interdépendance. En effet, la nation, la société civile et l'État ne sont pas simplement imbriqués, superposés, comme dans les compartiments étanches que seraient les aspects de la vie collective que chacun se subordonne. Ils sont en fait dépendants les uns des autres, l'épanouissement de la société devenant une condition de l'épanouissement de la nation et de l'État, et ainsi de suite, chaque élément nouant avec les autres d'étroites relations d'interdépendance. L'économique, qui n'est pas à proprement parler, dans le schéma que nous proposons, une fonction culturelle, devient donc une condition essentielle à l'épanouissement de la nation ${ }^{20}$. De même, le politique (l'État) a le devoir de favoriser le développement de la nationalité2 ${ }^{21}$. Ce principe d'interdépendance doit aussi s'appliquer aux relations qui unissent la cité terrestre et l'Église, le bien commun, finalité de la cité, devenant une condition du bien spirituel de l'homme, et vice 
versa. L'épanouissement national se trouve donc à être, de biais, un des fondements du bien spirituel.

En somme, l'individu en tant qu'être social appartient d'une part à une communauté spirituelle, et fait partie, d'autre part, d'une nation, d'une société, et relève d'un État, ce dans le cadre plus général d'une vie collective qui englobe chacune de ces entités. Et toutes, par le jeu d'interactions des aspects de la vie collective, convergent vers la réalisation de la fin spirituelle de l'homme, bien que ce n'en soit pas nécessairement la fin directe, immédiate.

\section{b) Définition de la nation ${ }^{22}$}

La nation comme telle, pour Minville, est un groupement qui se distingue d'abord par ses caractéristiques culturelles. On le constate aisément lorsqu'il parle de la nation canadienne-française et de son "double caractère» français et catholique ${ }^{23}$, qui sont pour lui les deux éléments les plus importants du patrimoine national ${ }^{24}$. Ce sont donc les traits culturels qui sont les marqueurs de la nation et c'est à eux que Minville se réfère en priorité lorsqu'il en décrit la nature.

Pour Minville, la nation est l'héritière d'une civilisation donnée, et les caractères que celle-ci lui lègue, qui en constituent l'âme, vont s'imposer aux nationaux comme une réalité incontournable à laquelle ils ne peuvent échapper, sous peine de connaitre un développement incomplet et faussé $e^{25}$. C'est l'exigence de la première orientation reçue, du «poids total des origines», comme disait Maurras que Minville cite ${ }^{26}$. La "discipline», la "culture de l'esprit ${ }^{27}$ » que le membre de la nation a hérité de la civilisation, est le «seul vrai fondement de sa personnalité». Même les aléas de l'histoire ou les spécificités du milieu ne peuvent les altérer ${ }^{28}$.

Il faut se garder toutefois de croire que l'histoire et le milieu ne sont que des contingences sans véritable influence sur le devenir de la nation. Bien au contraire, et c'est le deuxième volet de la définition de la nation que propose Minville, l'histoire et le milieu marquent de leur sceau le fragment de civilisation qui leur est exposé, le façonnent, le particularisent, bien qu'ils n'en altèrent pas les caractères fondamentaux. C'est ainsi que naissent les variantes d'une même civilisation ${ }^{29}$.

Bref, pour employer une métaphore qui s'accorde bien avec la vision organique qu'avait Minville de la réalité nationale, les nations sont comme des soeurs appartenant à une grande famille - une civilisation 
donnée. Chacune d'elles a été imprégnée des mêmes valeurs fondamentales, mais elles ont connu des cheminements de vie dans des conditions différentes, ce qui a contribué à les différencier. À noter cependant que les conditions et les circonstances sont entièrement responsables de la différenciation des nations, tandis qu'elles ne sont que partiellement responsables de la différenciation des soeurs, le reste étant assumé par les caractères et les aptitudes héritées à la naissance.

La nation, chez Minville, nous apparait en effet comme étant une sorte de «personne morale», d'organisme qui, à l'instar de l'indi$v^{\text {vidu }}{ }^{30}$, a ses propres caractères, ses propres aptitudes qu'elle se doit d'exercer dans un milieu qui lui est particulier et dont elle doit tenir compte en agissant. C'est par cette fidélité à elle-même, à la civilisation dont elle est issue, que la nation parviendra à la "plénitude de la vitalite ${ }^{31}$ »" et qu'elle atteindra des sommets de civilisation ne la laissant inférieure ni à elle-même, ni aux autres nations ${ }^{32}$.

Minville précise cependant que les collectivités, comme les individus d'ailleurs, ne sont pas livrées pieds et poings liés aux «lois d'un fatalisme sans merci», et que «c'est ce qui autorise à la fois les plus sombres inquiétudes et les plus hautes espérances ${ }^{32}$ ). Il est important de noter que, chez lui, aucun déterminisme ne parviendra jamais à éradiquer la part de liberté qui existe chez l'homme. Néanmoins, cette fidélité prend carrément figure d'une «rocation» de la nation, d'une sorte d'obligation historique envers les ancêtres, dont on continue l'oeuvre, et envers la civilisation, que chaque génération doit tâcher de transmettre dans son intégrité à la suivante. Tout ceci se trouve très bien exposé dans ce passage datant de 1930:

Rester fidèles à nous-mêmes dans un milieu modifié par le cours des événements; assurer la fructification totale du patrimoine reçu du passé, recueillir nos hérédités ethniques et psychologiques, les cultiver, les affiner; nous épanouir et non pas nous replier; nous adapter et non pas nous abandonner, tel est donc le devoir que l'histoire nous impose ${ }^{33}$.

On aura compris que la nation qui imite servilement les méthodes et les formes de vie d'une autre nation sans les passer au filtre de ses propres caractères et les adapter à sa situation ${ }^{34}$, est vouée à une sorte de médiocrité é et à n'être qu'un ersatz de la grande nation qui lui sert de modèle. C'est ainsi qu'il faut comprendre les mots d'ordre répétés de Minville à l'endroit de ses compatriotes, qu'il ne voulait pas voir devenir des «sous-Anglais» ou des "sous-Américains ${ }^{36}$ ", contre l'imitation bête des méthodes «anglo-saxonnes» dans quelque domaine que 
ce soit, mots d'ordre qu'on retrouvait notamment dans des articles au contenu percutant, voire polémique, tels «Les chocs en retour de l'anglomanie» et «Faisons comme les Anglais»" ${ }^{37}$.

En terminant, précisons que la nation, l'histoire et le milieu, pour Minville, ne programment pas l'individu comme s'il était un automate. Nous le répétons, l'individu conserve chez lui toujours une part de liberté inaliénable. Il n'en demeure pas moins que ces facteurs ont de lourdes conséquences psychologiques et morales, car ils lèguent à l'individu toute une manière de penser et $\mathrm{d}^{\prime}$ agir ${ }^{38}$. Le développement de la personne humaine est au coeur de l'oeuvre de Minville, et la richesse de l'héritage national ainsi que la fidélité à celui-ci en est certes une des conditions fondamentales. Il semble bien en effet que le développement de la nation soit pour lui davantage une condition du développement de la personne qu'un objectif en soi $^{39}$.

\section{c) Nationalisme économique}

L'expression «nationalisme économique» est, c'est le moins qu'on puisse dire, chargée d'équivoque. On l'emploie ici et là dans des sens très différents, parfois très restreints, parfois très larges. C'est qu'aucune définition du terme ne s'est réellement imposée jusqu'ici, sauf peut-être en ce qui a trait à la doctrine de ce nom qui avait cours au XIX $\mathrm{XIècle.}^{e}$ Malgré ces difficultés, nous tenterons ici de montrer que le nationalisme économique est une part essentielle de l'oeuvre de Minville.

Notons d'abord que Minville ne s'est jamais lui-même réclamé du nationalisme économique. Il emploie rarement le terme, et lorsqu'il le fait, il l'associe à la poussée de protectionnisme qu'a connu le monde occidental durant la crise économique des années trente, mouvement qu'il condamnait ${ }^{40}$. Ce qu'on retrouve chez lui, c'est donc un nationalisme économique qui ne dit pas son nom. Nous avons précisé, dans une sous-section précédente, la nature des liens qui unissent l'économique au national dans sa pensée. Rappelons brièvement l'essentiel de cette théorie: la nation comme telle se définit par ses caractères culturels spécifiques. Cependant, étant donné le jeu d'interactions des différents aspects de la vie collective, certains secteurs de l'activité humaine qui ne relèvent pas directement du culturel se trouvent à exercer une influence sur le devenir de la nation. C'est notamment le cas de l'économique, qui est ainsi, en quelque sorte, «nationalisé». Une politique économique nationaliste devra donc, si on suit le raisonnement de Minville, non seulement contribuer au développement culturel national, mais aussi s'inspirer de cette culture nationale, puisque c'est d'interdépendance qu'il 
parle. Voilà donc en quoi consiste l'essence du nationalisme économique de Minville. Cette idée est absolument fondamentale, car elle illustre parfaitement la conception qu'il se faisait de l'économique : c'est parce que celui-ci, du fait de ses liens avec les autres aspects de la vie collective, est ordonné à des fins supérieures, notamment à l'épanouissement de la nation, qu'il acquiert quelque valeur aux yeux de Minville. C'est précisément pour cette raison qu'il y consacrera les années les plus fécondes de sa vie.

Il nous faut cependant pousser l'analyse un peu plus loin. Le nationalisme économique est aussi le nom d'une doctrine qui fut développée au XIX ${ }^{e}$ siècle par des penseurs comme Friedrich List et Henry C. Carey, une doctrine qui, selon Kevin $\mathrm{Henley}^{41}$ et Paul Hugon ${ }^{42}$, se présente comme un mouvement prenant le contre-pied à la fois du libéralisme économique et du marxisme. Le nationalisme économique, pris dans ce sens précis, ne constitue pas l'influence dominante de l'oeuvre de Minville, ce privilège revenant à la doctrine sociale de l'Église, mais il la teinte néanmoins de ses thèmes et de ses conclusions. Il est de bon ton de rappeler ici que Minville avait très tôt été mis en contact avec la pensée de List, comme l'atteste le long article qu'il a publié à ce sujet dans la Revue trimestrielle canadienne en $1924^{43}$.

Dans sa thèse de doctorat, Henley relève quelques constantes de l'oeuvre de quatre penseurs (List, Carey, Charles Dupin et John B. Byles) qui seraient selon lui les dominantes du discours de l'École du nationalisme économique. Parmi ces éléments de doctrine, trois en particulier trouvent un écho dans l'oeuvre de Minville. Premièrement, l'idée que le progrès économique va de pair avec le progrès moral et intellectuel. N'est-ce pas là l'essence du principe de l'interdépendance des aspects de la vie collective? Deuxièmement, le recours suggéré à l'État pour contrer les effets pervers du régime de la libre entreprise. Minville prônait un semblable recours à l'intervention étatique, pour des raisons similaires. Finalement, l'idée que le progrès national exige le développement équilibré de la nation et des régions qui la composent, et ce dans tous les aspects de la vie économique, autant industriel qu'agricole ou commercial $^{44}$. Ces notions de développement équilibré, de développement régional et de développement intégral, on les retrouve chez Minville, comme nous aurons l'occasion de le constater.

Si nous rapprochons la pensée de Minville de celle de List, la parenté se précise et on en découvre d'autres aspects. D'abord, on l'aura deviné, List et Minville se rejoignent sur le plan du nationalisme ainsi que par leur commune opposition à l'individualisme libéral. Pour l'un 
comme pour l'autre en effet, l'homme abstrait extrait de son milieu social est une aberration, et une telle vision doit être condamnée $e^{45}$.

Ensuite, le principe de l'interdépendance des "forces productives» chez List est certainement à rapprocher de l'idée de l'interdépendance des aspects de la vie collective chez Minville. Les forces productives, pour List, sont le moteur du développement d'une nation. Elles englobent les éléments matériels (ressources naturelles, travail) ou immatériels (forces intellectuelles et sociales) qui ont une incidence sur l'accroissement de la production, sur l'augmentation du niveau culturel et moral, bref, sur l'accroissement de la puissance de la nation ${ }^{46}$. En somme, List nous dit simplement que c'est le potentiel, notamment intellectuel et institutionnel, de creer, d'accumuler et de conserver les richesses, qui conditionne le degré de puissance de celle-ci. Les forces productives se trouvent donc à agir les unes sur les autres, et c'est ainsi que List, un peu comme Minville, mais sans doute dans un esprit différent, est amené à lier l'économique stricto sensu aux autres aspects de la vie collective.

La pensée de Minville et celle de List se rejoignent également, de façon générale, sur la place et le rôle que doit tenir l'État. Dans un cas comme dans l'autre, on parle d'un Etat supplétif qui doit laisser s'épanouir l'initiative individuelle, mais qui est néanmoins investi d'un rôle interventionniste primordial. En substance, l'État doit d'une part contrôler dans une certaine mesure l'activité individuelle, afin de contrer les effets pervers du marché libre laissé à lui-même, et d'autre part jouer le rôle de chef d'orchestre du développement économique de la nation. Pour ce faire, il doit coordonner les activités individuelles et assurer la prévision du développement à long terme, en se chargeant notamment de la mise sur pied des infrastructures nécessaires ${ }^{47}$. L'un comme l'autre s'opposent donc à la vision minimaliste du rôle de l'État qui est celle du libéralisme économique classique.

Finalement, le dernier point commun des oeuvres de List et de Minville pourrait bien être la nécessité, perçue par l'un et l'autre, d'adapter la politique économique de la nation à son niveau de développement. List élabore en effet toute une théorie du développement économique suivant le principe de l'évolution par stades, c'est-à-dire que le développement d'une nation consiste précisément dans le passage d'un niveau à un autre, du stade primitif au stade agricole, etc, jusqu'au dernier stade, agricole, industriel et commercial. Les conditions et les moyens différant d'une étape à l'autre, il est normal que la politique économique de la nation soit adaptée à sa situation particulière. C'est pourquoi 
List privilégiera une politique protectionniste modérée pour les nations industrielles émergentes afin de soustraire la production nationale à la concurrence forcément intenable des nations plus avancées, et ce, jusqu'à ce que la nation parvienne au dernier stade de développement ${ }^{48}$. Il nous semble que Minville ne dit pas autre chose lorsqu'il réclame une politique économique québécoise adaptée non seulement aux caractères de la nation canadienne-française, mais également à sa situation. Il écrira par exemple que le Canada français, dépourvu de capitaux et de traditions commerciales, n'est pas encore assez fort, à son époque, pour se lancer dans la grande industrie. Par conséquent, il doit commencer par se construire un organisme économique basé sur la petite et la moyenne entreprise et en marge de l'organisation existante ${ }^{49}$.

\section{Programme de restauration sociale, économique et nationale}

Ces quelques précisions théoriques étant faites, nous allons maintenant présenter les solutions concrètes proposées par Minville en vue d'améliorer le sort de la nation canadienne-française. Minville observe la situation économique du Québec et du Canada français et constate qu'on a procédé à un développement industriel trop rapide, improvisé et inadapté à la situation et aux caractères de la nation. Le résultat en fut une excessive centralisation géographique et industrielle autour de Montréal et le dépérissement corrélatif des régions, autrement dit une rupture de l'équilibre entre la ville et la campagne ${ }^{50}$. Ceci a eu de profondes conséquences tant sur le plan social - chômage urbain, risque de désordre social - que sur le plan national - prolétarisation ${ }^{51}$ et dénationalisation (acculturation) du Canada français ${ }^{52}$. Minville s'attellera donc à la tâche d'élaborer un vaste programme de réformes comprenant, outre un volet concernant l'éducation nationale, un projet de développement économique rationnel et planifié, dans le but d'assurer le bien commun de la société tout entière et l'épanouissement de la nationalité canadienne-française. L'essentiel de ce programme de réformes économiques consiste en une triple décentralisation : premièrement, une décentralisation démographique, deuxièmement, une décentralisation de l'activité économique, et troisièmement, une décentralisation sociale et étatique. Voyons donc de quelle façon on devrait réaliser ces trois décentralisations selon Minville.

\section{a) Développement régional}

C'est par un vaste programme de restauration rurale et de développement régional que Minville entend opérer les deux premières décentralisations. Selon lui en effet, le problème fondamental est qu'au 
Québec, on a adopté une politique de développement économique par la grande industrie au détriment du monde rural et des régions. Ceci a eu pour résultat de provoquer une concentration excessive de l'activité économique, qui a à son tour causé un exode rural massif vers les États-Unis et vers la ville, donc le phénomène de concentration excessive de la population ${ }^{53}$. Cette restauration rurale doit avoir pour objectif d'augmenter le niveau de vie de la population rurale afin d'enrayer le mouvement de population de la campagne vers la ville, et ce tout en étant adaptée à la situation de la nation canadienne-française, qui ne dispose ni de capitaux suffisants, ni de traditions commerciales, de façon à favoriser son épanouissement tout en en construisant l'organisme économique sur des bases solides.

Puisque ce développement économique doit être méthodique et rationnel, l'étape préliminaire sera de faire l'inventaire complet des ressources du territoire, des marchés, et de procéder à des recherches scientifiques. En somme, il s'agit d'asseoir le programme de restauration économique sur une connaissance approfondie du milieu afin de pouvoir l'exploiter méthodiquement ${ }^{54}$.

Il faudrait ensuite mettre en branle une politique de colonisation méthodique, conçue non pas comme un simple palliatif à une situation économique difficile, mais comme une politique permanente et nécessaire ${ }^{55}$. Minville prend soin de préciser qu'il parle de colonisation méthodique adaptée aux besoins de l'heure, et qu'il ne s'agit donc pas, comme il le dit, de "[s] $\mathrm{s}$ armer de haches et de pioches et de [s"]installer à la lisière de la forêt ${ }^{5} \%$. La colonisation telle qu'il l'entend suppose une participation importante de l'État, qui doit, par exemple, mettre les terres à la disposition des colons, développer les infrastructures routières, établir un plan de colonisation, assister les colons en offrant, notamment, des primes au travail ${ }^{57}$. Voilà donc pour l'effort de décentralisation démographique.

Plus encore, une restauration rurale réussie implique également qu'une telle politique de colonisation s'accompagne d'un réel effort pour rendre la vie rurale au moins aussi attrayante sur le plan économique que peut l'être la vie d'ouvrier industriel. Dans un premier temps, il faut donc réformer l'agriculture, soit moderniser les méthodes, diversifier et spécialiser les cultures ${ }^{58}$, dans le cadre d'une agriculture de type familial $^{59}$. L'agriculture, élément stable de la vie économique ${ }^{60}$ et principale ressource contrôlée par les Canadiens français ${ }^{61}$, doit devenir la base inébranlable de leur organisme économique ainsi que leur tremplin vers l'industrie, ce pourquoi elle doit prospérer ${ }^{62}$. 
Dans un second temps, Minville propose d'apporter les correctifs nécessaires afin d'articuler l'exploitation des ressources naturelles du territoire, notamment de la forêt, à celle de l'agriculture. Au gré des différentes situations des régions et en fonction des données recueillies au cours de l'inventaire, on pourrait adopter une forme de colonisation mi-agricole, mi-forestière, ou encore qui combinerait la pêche et l'agriculture $^{63}$. Une telle solution assurerait aux exploitants un revenu à longueur d'année ${ }^{64}$ et permettrait de stabiliser la vie économique du monde rural et des régions par l'appoint d'une exploitation forestière profitant enfin à la population locale, contrairement au système du chantier et des grandes compagnies ${ }^{65}$. Il s'agit en somme d'harmoniser l'exploitation de toutes les ressources d'une région donnée en vue du développement de celle-ci et de la prospérité de sa population.

Dans un troisième temps, puisque l'activité économique ne se résume pas à l'agriculture, il faut ajouter un volet industriel pour que le programme de restauration rurale soit complet. Selon Minville, le type d'industrie qui convient le mieux à la population canadienne-française, sans capitaux ni traditions commerciales comme nous l'avons dit, est la petite et la moyenne industrie ${ }^{66}$. Il suggère donc de mettre sur pied tout un réseau de petites et de moyennes entreprises dans les régions québécoises en le finançant entre autres par l'épargne ${ }^{67}$. Ces industries susciteraient l'émergence de petites villes ${ }^{68}$ qui formeraient autant de centres industriels régionaux ${ }^{69}$, de petits foyers d'activité économique qu'en langage savant on appellerait probablement «pôles de développement». Ces petits centres industriels pourraient compléter le programme de développement économique en fournissant un débouché aux matières premières, notamment agricoles, exploitées dans la région qu'ils desservent, soit en les consommant, soit en les transformant en produits finis $^{70}$.

On pourrait également introduire dans ce plan la formule coopérative $^{71}$, conçue comme un moyen, d'une part, de démocratiser la gestion de l'entreprise ${ }^{72}$, et, d'autre part, de stabiliser le régime capitaliste en atténuant les conflits de classe grâce à la participation des travailleurs au profit ${ }^{73}$. Voilà donc comment, selon Minville, on pourrait opérer la décentralisation de l'activité économique qu'il souhaite tant. Le cas échéant, d'ici quelques générations, les Canadiens français, ayant grâce à ce programme acquis les capitaux et les traditions commerciales qui leur font défaut, pourraient se lancer à la reconquête de Montréal et de la grande industrie ${ }^{74}$. En ce sens, on peut qualifier la pensée de Minville d'uétapiste ${ }^{75}$ \%. 


\section{b) Corporatisme}

Pour compléter son programme de réformes, Minville y ajoute une troisième décentralisation, sociale et étatique cette fois-ci, qu'on opérerait via l'organisation corporative. C'est plus ou moins à partir de la promulgation de l'encyclique Quadragesimo Anno, en 1931, que Minville intègre le corporatisme à son programme. On ne sera donc pas étonné d'apprendre que le soutien qu'il apporte à cette institution est basé sur quelques-uns des préceptes chrétiens fondamentaux en matière sociale. Tout d'abord, l'idée de responsabilité de l'individu, tant envers la société qu'envers lui-même ${ }^{76}$, c'est-à-dire la responsabilité d'assurer l'épanouissement de toutes ses facultés, la responsabilité que suppose l'existence de la liberté de l'homme. Ensuite le principe de subsidiarité, étroitement lié à l'idée de responsabilité. Finalement, la collaboration des classes en vue du bien commun de l'ensemble de la sociétét7.

Il faut d'abord noter que Minville adhère au corporatisme dit social, qui émane du corps social lui-même, résultat d'une éducation sociale et nationale de la population et non d'une contrainte étatique, comme c'est le cas par exemple du corporatisme politique à l'italienne ${ }^{78}$. Un tel corporatisme est conçu comme un organisme intermédiaire entre l'État et l'entreprise privé ${ }^{79}$ et prend la forme d'un syndicalisme pousséso. Toute la population se regrouperait en associations correspondant à chaque branche d'activité (finance, agriculture, artisanat, etc.): ce sont les corporations. Celles-ci réuniraient, sur un pied d'égalité, patrons et ouvriers en un "conseil supérieun) de la profession où on en débattrait des problèmes ${ }^{81}$. Une fois constituée, la corporation se verrait reconnaître son existence juridique par l'État et deviendrait alors un organisme de droit public ${ }^{82}$. A partir de ce moment, les décisions prises par l'autorité professionnelle deviendraient exécutoires pour l'ensemble de la profession dans les domaines de juridiction économiques et sociaux qui lui ont été spécialement attribués, c'est-à-dire principalement l'organisation du travail et le contrôle de la production ${ }^{83}$. La corporation déchargerait ainsi l'État du très lourd fardeau des tâches qu'on a alors tendance à lui confier en ces années de crise, qui, selon Minville, ne sont pas de sa compétence. De cette façon, l'État pourrait se consacrer entièrement a ses propres fonctions ${ }^{84}$. Minville conçoit en effet le rôle de l'État comme étant supplétif, ce qui ne diminue par l'importance de ses tâches, bien au contraire, car il doit par exemple orienter le développement économique, stimuler et coordonner l'activité économique et sociale, assister la population dans le besoin par des programmes de travaux, etc. ${ }^{85}$ Voilà ce qui nous a amené à parler de décentralisation sociale et de décentralisation étatique. 
Ajoutons en terminant que, pour Minville, l'organisation corporative doit avoir une double finalité sociale et nationale. C'est-à-dire que, par elle, on doit concerter les énergies individuelles afin de dicter la politique qui doit à la fois assurer le bien commun de l'ensemble de la société et l'épanouissement de la nation canadienne-française ${ }^{86}$. C'est entre autres pourquoi, selon Minville, on devrait d'abord viser à instaurer le corporatisme sur le plan national canadien-français ${ }^{87}$.

\section{$* * *$}

Quelles conclusions peut-on tirer de tout ceci? En premier lieu, il faut noter que le grand programme de réformes d'Esdras Minville n'a jamais été appliqué dans son intégralité. Difficile dans ces conditions, évidemment, de savoir si les résultats de ces réformes auraient été ceux attendus. Toutefois, cela ne nous empêche pas de constater qu'on a là affaire à tout un système qui privilégie une action sur les structures (corporatisme, réforme de l'organisme économique) à une action qui serait plutôt de type conjoncturel (égislation sociale d'appoint), comme le keynésianisme, rejeté par Minville.

En second lieu, nous avons voulu, dans cet article, mettre l'accent sur les liens qui unissent le nationalisme à l'économique dans la pensée de Minville. Ce faisant, notre objectif était de montrer que les milieux intellectuels résolument traditionalistes sur les plans philosophique et spirituel que Linteau, Durocher et Robert appellent «clériconationalistes ${ }^{88}$, étaient loin d'être nécessairement réfractaires à toute forme de modernisation économique et sociale, et qu'il pouvait en émerger une pensée économique rigoureuse et rationnelle ainsi qu'une pensée réformatrice d'un radicalisme certain. Esdras Minville nous en fournit la démonstration de façon probante, et nous ajouterions même que l'oeuvre de la vie de Minville fut de tenter d'adapter la pensée traditionnelle chrétienne et nationaliste dont il se réclamait aux formes de la vie moderne. C'est d'ailleurs certainement là une bonne part de l'intérêt et de l'originalité que présente son cheminement intellectuel.

Deux motifs principaux nous autorisent à qualifier de «rigoureuse» et de «rationnelle» la politique économique de Minville. D'une part, un économiste de la renommée de François Perroux développera, au cours de sa carrière, des thèses qui, bien que plus systématiques, ne s'en approchent pas moins beaucoup de celles de Minville. D'ailleurs, si on en croit François-Albert Angers, Perroux lui-même aurait vu en Minville un précurseur de la "nouvelle économie», ou de l'économie du développement et de la planification ${ }^{89}$. D'autre part, un 
lien existe entre la pensée de Minville et les projets de planification du développement économique élaborés au Québec durant les années soixante. Ceux-ci s'inspiraient plus ou moins des mêmes constats et on y retrouvait certains des éléments essentiels du projet de réforme de Minville, comme le Conseil d'orientation économique et l'inventaire des ressources naturelles. Cependant, l'esprit qui animait les deux projets était passablement différent. Minville était un ruraliste "optimiste» qui aurait tenté de revitaliser sur le plan économique chaque petite bourgade. À l'opposé, l'entreprise des années soixante, beaucoup plus technicienne, prévoyait des déplacements de populations et la fermeture de certains villages gaspésiens, ce qui aurait été tout à fait inconcevable pour Minville. Le projet de ce dernier était éminemment respectueux du milieu humain, comme l'ensemble de son oeuvre d'ailleurs, ce qui n'était peutêtre pas le cas au même degré du programme des années soixante.

\section{NOTES}

' Cet article est une synthèse de deux textes que nous avons rédigés dans le cadre de nos études de maitrise à l'Université de Montréal, soit celui de notre communication donnée au colloque (Les intellectuels et les années trente : un brassage idéologique», du Congrès de l'Association canadienne-française pour l'avancement des sciences (ACFAS), le 15 mai 2000, et le premier chapitre de notre mémoire de maitrise en cours de rédaction, Esdras Minville, le catholicisme social et le nationalisme économique, 1923-1939, chapitre intitule «Notions fondamentales». Remerciements au professeur Pierre Trépanier pour ses précieux conseils et son soutien.

${ }^{2}$ Esdras Minville, Invitation à l'étude, Montréal, Fides, [1943], 171 p.

${ }^{3}$ Idem, «L'éducation économique», L'Action canadienne-franfaise, mai 1928, p. 263.

${ }^{4}$ Lionel Groulx, «L'oeuvre d'Esdras Minville», L'Action nationale, vol. 25 (janvier 1945), p. 11.

${ }^{5}$ Esdras Minville, «L'éducation économique», loc. cit., p. 264.

'Idem, [L'action catholique et l'économique], Conférence donnée entre 1936 et 1939, p.

7. Archives de l'École des Hautes Études commerciales (HEC), Fonds François-

Albert-Angers, P027T990014, Cahier des inédits datés.

'Esdras Minville, «L'éducation économique», loc. cit., p. 264.

${ }^{8}$ Idem, La croisée des chemins, Conférence donnée à Joliette, vers 1932-33, p. 8.

Archives HEC, Fonds François-Albert-Angers, P027T990014, Cahier des inédits datés.

${ }^{9}$ Idem, [L'action catbolique et l'économique], loc. cit., p. 4.

${ }^{10}$ Le bien commun est d'ensemble des conditions de vie sociale qui permettent aux hommes, aux familles et aux groupements de s'accomplir plus complètement et plus facilements. Encyclique Gaudium et spes, citée par Jean-Luc Chabot, La Doctrine sociale de $/$ Église, Paris, Presses universitaires de France, (coll. "Que sais-jeß») n 2495), 1992, p. 79.

${ }^{11}$ Esdras Minville, "L'éducation économique», loc. cit., p. 266.

${ }^{12}$ Voici la définition que donne Jean-Louis Quermonne de la société civile : «L'ensemble des rapports interindividuels, des structures familiales, sociales, économiques, cul- 
turelles, religieuses, qui se déploient dans une société donnée, en dehors du cadre et de l'intervention de l'État.» Cité par Marc Chevrier, «La société civile, l'État subsidiaire et la responsabilité civique au Québec), Conférence prononcée au colloque du Ralliement québécois, 30 octobre 1999. Le texte est disponible sur la toile, à l'adresse suivante : www.agora.qc.ca.

${ }^{13} \mathrm{La}$ nation, pour Minville, est un groupement qui se distingue d'abord par ses caractéristiques culturelles. Nous y reviendrons.

${ }^{14}$ Esdras Minville, [Solidarité nationale], Discours de la Saint-Jean-Baptiste, 24 juin 1933, p. 12. Archives HEC, Fonds François-Albert-Angers, P027T990014, Cahicr des inédits datés.

15 Idem, "La bourgeoisie et l'économique», dans L'avenir de notre bourgeoisie, Montréal, Éditions de la JIC et Éditions Bernard Valiquette, 1939, p. 55.

${ }^{16} \mathrm{Idem}$, «L'éducation économique», loc. cit., p. 266.

${ }^{17}$ Idem, [L'éducation nationale], Discours à la Société des Conférences d'Ottawa, 9 février 1936, p. 9. Archives HEC, Fonds François-Albert-Angers, P027T990014, Cahier des inédits datés.

${ }^{18}$ Idem, (sous le pseudonyme de Jacques Dumont), «Méditation pour jeunes politiques", L'Action frangaise, janvier 1927, p. 30.

${ }^{19}$ Voir, par exemple, Esdras Minville, "Ce que nous voulons", L'Action nationale, octobre 1935, p. 99; [Solidarité nationale], loc. cit., p. 12.

${ }^{20} I d e m$, [Le problème national, Conférence prononcée devant la Société Saint-JeanBaptiste de Coaticook, 1930, p. 7. Archives HEC, Fonds François-Albert Angers, P027T990014, Cahier des inédits datés.

${ }^{21}$ Lettre d'Esdras Minville à Lionel Groulx, 30 juin 1927, 3 p. dactylographiées. Archives du Centre de recherche Lionel-Groulx (ACRLG), Fonds Lionel-Groulx, Correspondance Groulx-Minville, P1/A, dossier 2714.

${ }^{22}$ À cette époque, Minville emploie généralement les termes de upeuple» ou de «race» pour désigner la nation.

${ }^{23}$ Esdras Minville, [Le probleme national, loc. cit., p. 2.

${ }^{24}$ Idem, «Discours de M. Minville», L'Action nationale, février 1933, p. 123.

${ }^{25}$ Idem, «L'éducation économique», loc. cit., p. 285; [L'éducation nationale], loc. cit., p. 4.

${ }^{20} \mathrm{Idem}$, [Le probleme national, loc. cit., p. 3.

${ }^{27}$ Idem, «L'éducation économique», loc. cit., p. 285.

${ }^{28}$ Idem, [L'éducation nationale], loc. cit., p. 4.

${ }^{29}$ Idem, «L'éducation économique», loc. cit., p. 285.

${ }^{30}$ Esdras Minville fait cette comparaison dans Instruction ou éducation? $A$ propos de l'enseignement secondaire, Montréal, Brochures de l'École sociale populaire (ESP) $\mathrm{n}^{\text {os }} 204-$ 205, 1931, p. 46.

${ }^{31}$ Esdras Minville, [L'éducation nationale], loc. cit., p. 4.

${ }^{32}$ Idem, uLes chocs en retour de l'anglomanie», L'Action nationale, avril 1934, p. 198.

${ }^{32}$ Idem, [Le probleme national, loc. cit., p. 2.

${ }^{33}$ Ibid., p. 3.

${ }^{34} \mathrm{Idem}$, «La bourgeoisie et l'économique», loc. cit., p. 31.

${ }^{35}$ Idem, «Les chocs en retour de l'anglomanie», loc. cit., p. 198.

${ }^{36}$ Idem, La croisée des chemins, vers 1932-33, loc. cit., p. 7.

${ }^{37}$ Idem, (sous le pseudonyme de François Chantal), «Faisons comme les Anglais», 
L'Action nationale, Premier semestre 1934, p. 346-356.

${ }^{38} \mathrm{Idem}$, «Les chocs en retour de l'anglomanie», loc. cit., p. 196-197.

${ }^{39}$ Ibid., p. 198.

${ }^{40}$ Idem, La croisée des chemins, vers 1932-33, loc. cit., p. 5.

${ }^{41}$ Kevin Henley, La doctrine du nationalisme économique au Canada central, 1846-1885, Thèse de Ph.D. (Histoire), Université du Québec à Montréal, 1990, 408 p.

${ }^{42}$ Paul Hugon, Les doctrines économiques, Montréal, Fides, [1945], 413 p.

${ }^{43}$ Esdras Minville, "L'économie nationale», Revue trimestrielle canadienne, $\mathrm{n}^{\circ} 10$ (mars 1924), p. 49-78.

${ }^{44}$ Henley, op. cit., p. 93-94.

${ }^{45}$ Monique Anson-Meyer, Un économiste du développement au XIX' siècle : Friedrich List, Grenoble, Presses universitaires de Grenoble, 1982, p. 42; Minville, «La juste répartition des richesses", dans L'ordre social chrétien : compte rendu des cours et conférences, Montréal, Semaines sociales du Canada, ESP, 1932, p. 65.

${ }^{46}$ Anson-Meyer, op cit., p. 66.

${ }^{47}$ Ibid., p. 137-147.

${ }^{48}$ Ibid., p. 95-103.

${ }^{49}$ Esdras Minville, «Agir pour vivre!), L'Actualité économique, vol. $3, \mathrm{n}^{\circ} 8$ (novembre 1927), p. 154; La croisée des chemins, vers 1932-33, loc. cit., p. 7 et 23.

${ }^{50}$ Idem, «Le capital étrangen», L'Action française, juin 1924, p. 330; «Agir pour

vivre!), loc. cit., p. 151.

${ }^{51} \mathrm{Idem}$, "Agir pour vivre!», loc. cit., p. 153.

52 Idem, «Le capital étrangen, loc. cit., p. 341.

${ }^{53}$ Idem, "Agir pour vivre!), loc. cit., p. 148-151.

${ }^{54} \mathrm{Idem}$, «Une idée ancienne toujours opportune», L'Actualité économique, vol. 11-I, n ${ }^{\text {os }}$ 3-4 (juin-juillet 1935), p. 259-263.

${ }^{55}$ Idem, L'oeuvre de la colonisation, Montréal, Brochure de l'ESP n ${ }^{\circ} 238,1933$, p. 8.

${ }^{56}$ Idem, La croisée des chemins, vers 1932-33, loc. cit., p. 15.

${ }^{57}$ Idem, L'oeuvre de la colonisation, loc. cit., p. 16-22.

${ }^{58}$ Idem, La croisée des chemins, vers 1932-33, loc. cit., p. 17-18.

${ }^{59}$ Lettre d'Esdras Minville à Eugène L'Heureux, 21 décembre 1932. Archives de l'École des HEC, Fonds du Directorat, A007Z990010, dossier 141.

${ }^{60}$ Esdras Minville, L'oewure de la colonisation, loc. cit., p. 9.

${ }^{61}$ Idem, «Agir pour vivrel», loc. cit., p. 154.

${ }^{62} \mathrm{Idem}$, «Le capital étrangen, loc. cit., p. 346.

${ }^{63} \mathrm{Idem}$, "Quelques aspects du problème social dans la province de Québec», L'Actualité économique, vol. 14-I, $\mathrm{n}^{\circ} 7$ (octobre 1938), p. 412-413.

${ }^{64} \mathrm{Idem}$, «La bourgeoisie et l'économique», loc. cit., p. 49.

${ }^{65} \mathrm{Idem}$, "Quelques aspects du problème social...", loc. cit., p. 413-416.

${ }^{6} \mathrm{Idem}$, «Pourquoi de la petite industrie», L'Actualité économique, vol. 9, $\mathrm{n}^{\text {os }} 3-4$ (juinjuillet 1933), p. 169-170.

${ }^{67} \mathrm{Idem}$, «L'organisation de l'épargne», L'Actualité économique, vol. $10, \mathrm{n}^{\circ} 9$ (décembre 1934), p. 503.

${ }^{68}$ Idem, La croisée des chemins, vers 1932-33, loc. cit., p. 22.

${ }^{69} \mathrm{Idem}$, "Une idée ancienne toujours opportune», loc. cit., p. 262.

${ }^{70} \mathrm{Idem}$, «Pourquoi de la petite industrie», loc. cit., p. 172.

${ }^{71}$ Idem, «Le capital étrangen", loc. cit., p. 347-348. 
72 Esdras Minville et François Vézina, "De Montréal à Victoria III», L'Actualité économique, vol. $5, \mathrm{n}^{\circ} 11$ (février 1930), p. 329.

${ }^{73}$ Esdras Minville, «Libéralisme? Communisme? Corporatisme?», L'Actualité économique, vol. 12-II, n² 2 (décembre 1936), p. 165.

${ }^{74}$ Idem, La croisée des chemins, vers 1932-33, loc. cit., p. 23.

${ }^{75}$ Idem, La politique qu'il nous faut, Montréal, Association catholique de la Jeunesse canadienne-française (ACJC), 1932, p. 7.

${ }^{76} \mathrm{Idem}$, «Libéralisme? Communisme? Corporatisme?», loc. cit., p. 162.

${ }^{77}$ Idem, "Le syndicalisme catholique», L'Actualité économique, vol. 13-I, $\mathrm{n}^{\circ} 7$ (octobre 1937), p. 471.

${ }^{78}$ Idem, [Le corporatisme], causerie, vers 1937, p. 5. Archives HEC, Fonds FrançoisAlbert-Angers, P027T990014, Cahier des inédits.

${ }^{79} \mathrm{Idem}$, «L'institution corporative», L'Actualité économique, vol. 11-I, $\mathrm{n}^{\text {os }}$ 5-6 (aoûtseptembre 1935), p. 380.

${ }^{80}$ Idem, «L'organisation corporative en Belgique», L'Actualité économique, vol. 11-II, $\mathrm{n}^{\circ} 1$ (novembre 1935), p. 72.

${ }^{81} \mathrm{Idem}$, "Comment établir l'organisation corporatiste au Canada), dans L'organisation professionnelle : compte rendu des cours et conférences, Semaines sociales du Canada, ESP, 1936, p. 254.

${ }^{82}$ Idem, [Le corporatisme], loc. cit., p. 5.

${ }^{83}$ Père Chagnon, cité par Minville, [Le corporatisme], loc. cit., p. 5-6.

${ }^{84}$ Esdras Minville, [Le corporatisme], loc. cit.,p. 7.

${ }^{85}$ Idem, «L'aide de l'État à l'industrie et au commerces, L'Actualité économique, vol. 13II, $\mathrm{n}^{\circ} 4$ (février 1938), p. 370; La législation ouvrière et le régime social dans la province de Québec, Appendice 5 du Rapport de la Commission Rowell-Sirois, Ottawa, J.O. Patenaude Imprimeur du Roi, 1939, p. 98.

${ }^{86} \mathrm{Idem}$, [Le corporatisme], loc. cit., p. 12.

${ }^{87} \mathrm{Idem}$, “Comment établir l'organisation...», loc. cit., p. 243.

${ }^{88}$ Paul-André Linteau, René Durocher et Jean-Claude Robert, Histoire du Québec contemporain, tome 1, De la Confédération à la crise (1867-1929), Montréal, Boréal, 1989, p. 700-707.

${ }^{89}$ François-Albert Angers, "Préface» de L'économie du Québec et la science économique, Montréal, Fides et Presses HEC, 1979, p. 28. 